\title{
Modelling of mixed damage on fibre reinforced composite laminates subjected to low velocity impact
}

\author{
JL Curiel Sosa', S Phaneendra ${ }^{2}$ and J Munoz ${ }^{2}$
}

\begin{abstract}
This article presents a numerical technique for the computation of damage in fibre-reinforced laminated composites which is embedded into an explicit finite element method. The technique is composed of two main components. First, a novel characterisation of the directions in which the various modes of damage propagate, which produces an effective damage localisation. This is conducted taking into consideration the strain-rate dependence. Second, a new mapping between the strain and stress spaces for the computation of the damage surfaces whereby time-stepping convergence is enhanced. Additionally, new damage initiation criteria in terms of strain damage surfaces are presented. Details of the in-house code developed are presented as well as the programming features. The capabilities of the technique are shown by means of tests on single fibre-reinforced element and low velocity impact on the laminate. It is shown that delamination is located in the expected regions by gradual progression of damage internal variables.
\end{abstract}

\section{Keywords}

Damage, composite, finite element method, impact, laminate, failure

\section{Introduction}

From a computational point of view, numerical techniques based on a progressive evolution of 2 damage in composite materials, e.g. Maimi et al. (2007a, 2007b), Tan (1991), Harris et al. (1995), Raimondo et al. (2012), Donadon et al. (2008) and Liu and Zheng (2010), are more stable than stress failure criteria, e.g. Hashin (1980), Tsai and Wu (1971), Puck and Schurmann (1998) and Chang and Chang (1987), with respect to the erosion - deletion - of finite elements as these progressively degrade, matching stiffness deterioration and mitigating in that manner the undesirable instabilities of the numerical method. Advantages and disadvantages of these two general trends mentioned are

\footnotetext{
'Mechanical Engineering Subject Group, Department of Engineering and Mathematics, Sheffield Hallam University, UK

${ }^{2} \mathrm{LaCaN}$, Department of Applied Mathematics III, School of Civil Engineering, Polytechnic University of Catalonia, Spain

Corresponding author:

JL Cur iel Sosa, Mechanical Engineering Subject Group, Department of Engineering and Mathematics, Sheffield Hallam University. Howard Street, Sheffield, SI IWB, UK

Email: j.Lcuriel-sosa@shu.ac.uk
} 
still recurring subjects of debate (Daniel, 2007). Cohesive elements have become very popular as discontinuities may efficiently be simulated, e.g. splitting due to delamination (Camanho and Mathews, 1999; Camanho et al., 2003; Tvergaard, 2004; Iannucci and Willows, 2006). An up-todate review can be found in Wisnom (2010). The proposed technique admits linkage to cohesive elements, although this is not attempted at this time.

The proposed technique is framed on the thermodynamics of irreversible process following Chaboche (1992). Other interesting thermodynamical approaches for damage mechanics of composites are also found in literature, e.g. Simo and Ju (1987), Turon et al. (2006), amongst others. From a purely damage mechanics point of view, the main features of the proposed technique are the computation of paths of damages which provide an effective localisation of the different damage modes and, second, the computation of damage on the strain space through the generation of a mapping between the strain and stress spaces where the so-called normalised energy release rates are readily computed. Thus, the undamaged domain is defined on the strain space bounded for a set of quadratic damage surfaces. The approach is based on computing the damage at quadrature points, i.e. gauss points, within each finite element, forming the mesh of the composite which is performed in the strain space. Moreover, fracture energy - modes I and II - can be added to the model and mapped onto to the strain space following an analogous strategy. This last point permits to treat all the variables associated to failure on the composite in a progressive manner which provides higher stability and convergence in the explicit finite element method (XPFEM) procedure, as the removal of a finite element often causes oscillations when using XPFEM, creating instability, and, eventually, divergence (Camanho et al., 2001).

This article is outlined as follows. First, the thermodynamical background, in which the technique is framed, is briefly presented. Second, definition of the measurements of stress utilised are provided. Third, damage components of the technique are described. Fourth, the integration within XPFEM is presented in some detail. Finally, numerical results and discussion are provided. Additionally, an appendix containing relevant tensors is included.

\section{Thermodynamics of damage in dissipative materials}

The case of irreversible process in dissipative materials considered follows the framework by Chaboche (1992). A brief summary is highlighted in this section to frame the background of the study. The definition of the damage state is described by a set of internal damage variables (Germain et al., 1983; Desmorat et al., 2010; Yang et al., 2010). The thermodynamic potential is represented by the specific free energy, $\Phi$. Since non-metallic composites are approached, plasticity and hardening terms may be ignored. In this case, the free energy $\Phi$ takes the following expression

$$
\Phi:=\frac{1}{2} \varepsilon: \mathbf{C}: \boldsymbol{\varepsilon}
$$

Here and henceforth, bold symbols and bold characters denote tensor and vector variables. The symbol $\varepsilon$ denotes the second-order strain tensor and '?' the inner product. The constitutive law is represented by the damaged secant stiffness, $\mathbf{C}$, which is, in general, a fourth-order tensor (Simo and $\mathrm{Ju}, 1987)$. This tensor is a function of the damage internal variables $\eta_{i j}$, equation (2). These variables are integrated in the tensor $\boldsymbol{\eta}$.

$$
\mathbf{C}(\boldsymbol{\eta})=2 \frac{\partial \Phi}{\partial(\boldsymbol{\varepsilon} \otimes \boldsymbol{\varepsilon})} \quad \boldsymbol{\sigma}=\frac{\partial \Phi}{\partial \boldsymbol{\varepsilon}}=\mathbf{C}: \boldsymbol{\varepsilon}
$$


where $\otimes$ is the tensor product. Chaboche (1992) defines the energy release rates as the derivative of this potential respect to the internal damage variables,

$$
\mathbf{Y}(\boldsymbol{\varepsilon}, \boldsymbol{\eta}):=-\frac{\partial \Phi}{\partial \boldsymbol{\eta}}=-\frac{1}{2} \boldsymbol{\varepsilon}: \frac{\partial \mathbf{C}}{\partial \boldsymbol{\eta}}: \boldsymbol{\varepsilon}
$$

where $\mathbf{Y}$ denotes a second-order tensor containing the thermodynamic forces $Y_{i j}$. The dissipative potential is related to the irreversibility of the process which is caused by distinct damage modes in the case of composites. The dissipative potential is a function of the thermodynamic forces $Y_{i j}$ and the damage state itself. Following Chaboche (1992), the dissipative potential is replaced by damage criteria. Equation (4) defines the elastic undamaged domain on the strain space.

$$
g:=g(\boldsymbol{\varepsilon} \otimes \boldsymbol{\varepsilon}, \boldsymbol{\eta}) \leq 0
$$

In the proposed technique ('Damage computation'), a new criteria is proposed as a substitute for the dissipative potential which takes into account the so-called normalised energy release rates as constituents.

\section{Measurement of stress}

Although the stress-strain relationship, in general, for composites with potential anisotropic damage implies the formulation in terms of a fourth-order tensor (Simo and $\mathrm{Ju}, 1987$ ), the attempt, here, is restricted to orthotropic fibre-reinforced laminae and, therefore, the constitutive tensor can be represented by a second-order tensor as described below. The stress is measured using the definition of effective stress $\hat{\boldsymbol{\sigma}}$ by Chaboche (1981), that is equation (5). In Figure 1, the different measurements of stress, effective and nominal, are schematically depicted. Furthermore, the principle of strain equivalence (Lemaitre and Chaboche, 1990) is applied on the constitutive law. Following Simo and Ju (1987), the damage matrix $\mathbf{D}$ is formed by the damage internal variables $\eta_{i j}$, equation (6), which are related to stiffness degradation of the composite in the corresponding directions.

$$
\hat{\boldsymbol{\sigma}}_{n}=\mathbf{D}\left(\boldsymbol{\eta}_{n}\right) \cdot \boldsymbol{\sigma}_{n}
$$
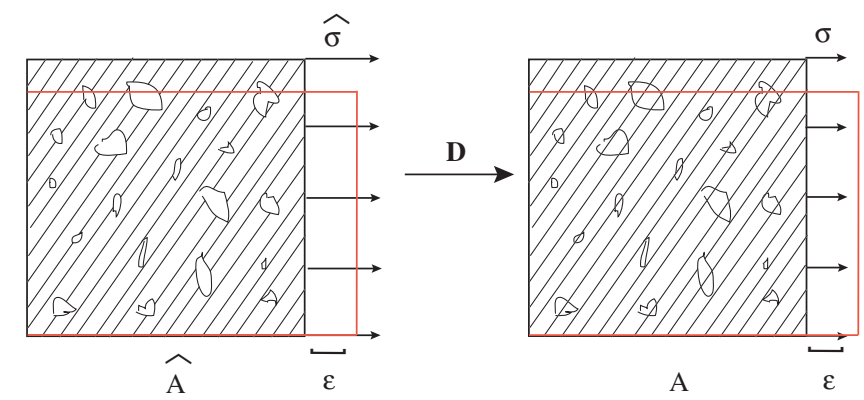

Figure I. Schematic representation of the principle of strain equivalence (Lemaitre and Chaboche, 1990) relating both measures of stress used, effective $\hat{\sigma}$ and nominal $\sigma$.

The red line indicates the final deformed configuration. This principle asserts that the effective stress acting over $\hat{A}$, total area minus area of the microcracks or defects, produces the same strain as that of the nominal stress acting over the total area $A$. 
Material axis $\{1,2,3\}$ are used for the definition of tensors and vectors at plies - unidirectional (UD) layers. They are local in the ply and are rotated to a global axis $\{x, y, z\}$ for the final coupling of the global system of momentum equations. The transformation of tensors from an off-axis configuration can be consulted in any standard reference for mechanics of laminar composites such as Staab (1999). Material axis at the ply level are defined following the conventional standards:

- 1-direction: longitudinal to fibres,

- 2-direction: in-plane perpendicular to fibres, and

- 3-direction: out-of-plane perpendicular to fibres.

The stress is introduced as a contracted vector, $\boldsymbol{\sigma}_{n}=\left[\sigma_{11}, \sigma_{22}, \sigma_{33}, \sigma_{12}, \sigma_{23}, \sigma_{31}\right]_{n}^{T}$, equation (5), and the diagonal of the damage tensor contains the damage variables $\eta_{i j}$ linked to the deterioration of the stiffness components, as shown in equation (6). $\boldsymbol{\eta}$ denotes a vector - from a contracted tensor containing the damage internal variables, $\boldsymbol{\eta}_{n}=\left[\eta_{11}, \eta_{22}, \eta_{33}, \eta_{12}, \eta_{23}, \eta_{31}\right]_{n}^{T}$, which define the damage state. Henceforth, subscript $n$ denotes that the computation of the variable or tensor affected by it refers to the time step $t_{n}$ of the proposed time-marching technique.

$$
D_{i j}\left(\boldsymbol{\eta}_{n}\right)=\delta_{i j} /\left(1-\left.\eta_{i j}\right|_{n}\right)
$$

where $\delta_{i j}$ is the Kronecker delta. ${ }^{\text {a }}$ The stress-strain relationship is provided in equation (7), where $\mathbf{C}_{0}$ is a second-order constitutive tensor that contains the initial values of stiffness components, unaffected by any damage. Finally, the stress-strain relationship may be written through a constitutive matrix that integrates the deterioration of its components due to the damage modes, equation (7).

$$
\boldsymbol{\sigma}_{n}=\mathbf{D}^{-1}\left(\boldsymbol{\eta}_{n}\right) \cdot \mathbf{C}_{\mathbf{0}} \cdot \boldsymbol{\varepsilon}_{n}=\mathbf{C}_{n} \cdot \boldsymbol{\varepsilon}_{n}
$$

\section{Damage computation}

\section{Mapping and characterisation of undamaged domain}

The damage surfaces bound the undamaged domain composed for as many as the numbers of damage modes $\xi$ modelled. In other words, every damage mode is linked to a stress damage surface that in turn is mapped onto the strain space as explained next. Normalised energy release rates are provided in equation (8). These strain damage surfaces are obtained by the mapping in equation (11), from normalised energy release rates on the stress space

$$
\bar{Y}_{i j}\left(\boldsymbol{\sigma}_{n}, \boldsymbol{\eta}_{n}\right):= \begin{cases}\left.\frac{\sigma_{i i}^{2}}{2 E_{i}\left(1-\eta_{i i}^{2}\right) X_{i i}^{(j)}}\right|_{n} & \text { if } i=j \text { and } \sigma_{i i} \geq 0 \\ \left.\frac{\sigma_{i i}^{2}}{2 E_{i}\left(1-\eta_{i j}^{2}\right) X_{i i}^{(c)}}\right|_{n} & \text { if } i=j \text { and } \sigma_{i i}<0 \\ \left.\frac{\sigma_{i j}^{2}}{2 S_{i j}\left(1-\eta_{i j}^{2}\right) X_{i j}}\right|_{n} & \text { if } i \neq j\end{cases}
$$

\footnotetext{
${ }^{\mathrm{a}}$ The Kronecker delta, $\delta_{i j}$, is I if $i=j$ and, 0 otherwise.
} 
where $E_{i}$ denotes the Young's modulus in direction $i, S_{i j}$ the elastic shear modulus associated to directions $i$ and $j, X_{i i}^{(t)}$ the direct tensile strength of the laminate in direction $i$ and $X_{i j}$ the shear strength related to the corresponding directions. The stress damage surfaces are

$$
f^{\xi}\left(\boldsymbol{\sigma}_{n}, \boldsymbol{\eta}_{n}\right):=f^{\xi}\left(\bar{Y}_{i j}\left(\boldsymbol{\sigma}_{n}, \boldsymbol{\eta}_{n}\right)\right) \quad \xi=1,2, \ldots, m
$$

For convenience, equation (9) is rearranged as follows in equation (10).

$$
f^{\xi}\left(\boldsymbol{\sigma}_{n}, \boldsymbol{\eta}_{n}\right):=\boldsymbol{\sigma}_{n}^{T} \cdot \mathbf{F}^{\xi}\left(\boldsymbol{\eta}_{n}\right) \cdot \boldsymbol{\sigma}_{n}-1 \quad \xi=1,2, \ldots, m
$$

where $\mathbf{F}^{\xi}$ denotes the tensor associated to the damage mode $\xi$ and $m$ the total number of damage modes modelled, see A. The mapping in equation (11) is obtained by previous computation of these damage surfaces on the stress space. Thus, these stress damage surfaces are built as function of the so-called normalised energy release rates in equation (8) due to the propagation of damage variables. The stress damage surfaces $f^{\xi}$ shrink with the progression and development of any damage mode affecting them. The initial spherical damage quadratic form turns into an ellipsoid (Figure (2)) due to the progression of damage and the volume is re-shaped and, therefore, the undamaged domain on the strain space is consequently reduced. Once $f^{\xi}$ is calculated, the corresponding $g^{\xi}$ damage surface on the strain space is computed at each quadrature point on the finite element under calculation according to

$$
\begin{gathered}
\mathbf{G}^{\xi}\left(\boldsymbol{\eta}_{n}\right)=\boldsymbol{C}_{n}^{T} \cdot \mathbf{F}^{\xi}\left(\boldsymbol{\eta}_{n}\right) \cdot \boldsymbol{C}_{n} \quad \xi=1,2, \ldots, m \\
g^{\xi}\left(\boldsymbol{\varepsilon}_{n}, \boldsymbol{\eta}_{n}\right):=\boldsymbol{\varepsilon}_{n}^{T} \cdot \mathbf{G}^{\xi}\left(\boldsymbol{\eta}_{n}\right) \cdot \boldsymbol{\varepsilon}_{n}-1 \quad \xi=1,2, \ldots, m
\end{gathered}
$$

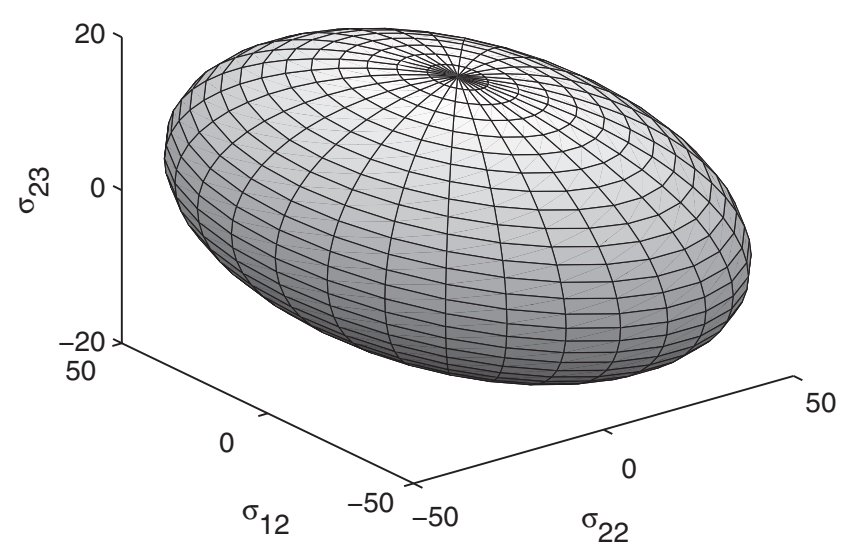

Figure 2. Stress damage surface due to fibre rupture $\left(\xi_{1}\right)$ on a tensile test. The initial spherical damage quadratic form turns into an ellipsoid due to the progression of damage. 
Remark: The technique permits the inclusion of fracture energy that conveniently can be used for the utilisation of cohesive elements in future works. The fracture energy in mode I for interply failure as defined by Johnson et al. (2001)

$$
\mathcal{G}_{I}:=\int_{0}^{u_{33}} \sigma_{33} \mathrm{~d} u_{33}
$$

is re-defined for readiness of computation at quadrature points as given by

$$
\mathcal{G}_{I}^{\xi}=\sum_{k=1}^{n q} \varsigma^{(k)} \sigma_{33}^{(k)} \varepsilon_{33}^{(k)}
$$

where the direction 3 is perpendicular to the interply plane. $\mathcal{G}_{I c}^{\xi}$ fracture toughness in mode I, $n q$ the number of quadrature points used for numerical integration of the expression of the energy release rate $\mathcal{G}_{I}^{\xi}$ and $\varsigma^{(\kappa)}$ the weight associated to the quadrature point. Analogously, a stress damage surface is generated that is mapped onto the strain space, equation (15). Then, this surface will form part of the boundary of the undamaged domain in the strain space. This represents a huge advantage as having all damage surfaces in the strain space bounding the undamaged domain, which is itself evolving in volume and shape, with the progression of the damage modes permits an straightforward computation of damage at every time step of XPFEM.

$$
f^{\xi}\left(\boldsymbol{\sigma}_{n}, \boldsymbol{\eta}_{n}\right)=\left(\frac{\mathcal{G}_{I}^{\xi}}{\mathcal{G}_{I c}^{\xi}}\right)^{p}-1
$$

\section{Criterion for progression of damage}

A new criterion is presented in this section for computing a progression of a particular damage mode at any time step. A damage mode is compued at time step $t_{n}$ if the criterion in equation (16) is satisfied, accumulating in that manner that specific damage mode. The derivation of the criterion is performed as follows:

- Application of the proposed mapping between stress and strain spaces to obtain the boundaries of the undamaged domain in the strain space (equation (12)) from the corresponding surface on the stress space (equation (10)).

- Accumulation of a specific damage mode occurs only if $\nabla_{\varepsilon} g_{n}^{\xi} \cdot \dot{\boldsymbol{\varepsilon}}_{n}>0$. Note that this is a direct condition necessary for the accumulation of the damage mode $\xi$ in the current time step $T_{n}$.

$$
\nabla_{\varepsilon} g_{n}^{\xi} \cdot \dot{\boldsymbol{\varepsilon}}_{n}>0 \quad g^{\xi}\left(\boldsymbol{\varepsilon}_{n}, \boldsymbol{\eta}_{n}\right) \geq 0 \quad \xi=1,2, \ldots, m
$$

From a mathematical point of view, it asserts that if the gradient of damage surface at the current state is pointing outwards with an angle less than 90 with the strain rate vector, then the computation, at current time step for that particular damage mode, is performed. In this manner, the strain rate dependence is taken into account which permits, for instance, distinction at different impact velocities. Further details of the procedure are depicted in the flowchart provided in Figure 3.

\section{Directions for propagation of damage}

The characterisation of directional vectors $\mathbf{d}^{\xi}$ is given by equation (17), which points to the damage mode direction - or path - to follow on the strain space.

$$
\mathbf{d}_{n}^{\xi}:=\boldsymbol{\varepsilon}_{n}^{T} \cdot\left(\mathbf{G}_{n}^{\xi T}+\mathbf{G}_{n}^{\xi}\right) /\left\|\nabla_{\varepsilon} g_{n}^{\xi}\right\| \quad \xi=1,2, \ldots, m
$$




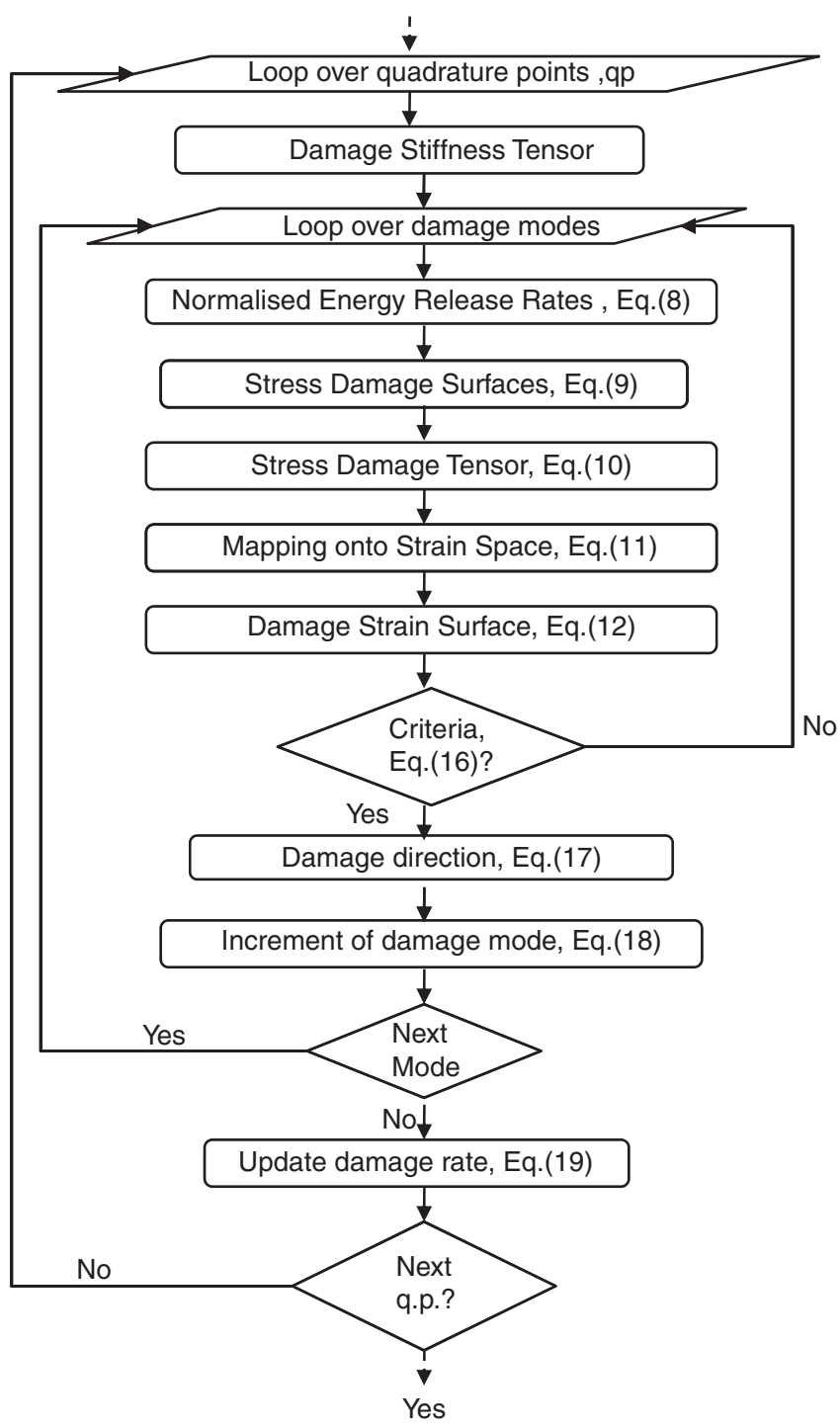

Figure 3. Detailed flowchart of the calculation of damage progression associated to the different modes computed at the quadrature point level.

This algorithm is nested within the loop over finite elements and embedded into the time-stepping scheme as described in 'Explicit time-stepping scheme'. Note that in XPFEM, there is a spatial discretisation by finite elements and a time discretisation by finite differences (Belytschko et al., 200I).

\section{Progression of damage}

Once the criterion in equation (16) is accomplished, the progression of the damage mode $\xi$ concerned is conducted by the description given by

$$
\psi_{n}^{\xi}:=\left(\nabla_{\varepsilon} g_{n}^{\xi} \cdot \dot{\boldsymbol{\varepsilon}}_{n}\right)^{1 / p} \quad \xi=1,2, \ldots, m
$$


where $\psi^{\xi}$ denotes the corresponding increment and $p$ a parameter depending upon the composite material and the geometry that must be adjusted for a particular problem. In general, $p=2$ produced excellent results, see 'Numerical results and discussion'. The square root operator was incorporated as preliminary results showed an excessive increment of the damage. Its inclusion proved to be more physically accurate respect to the degree of damage attained. The smoothness of the numerical process was also positively affected. Finally, the damage rate vector is computed at the current time step as given by equation (19) following Matzenmiller et al. (1995). In this case, the relation, in terms of growth coefficients, becomes non-linear due to the re-definition of $\psi^{\xi}$.

$$
\dot{\boldsymbol{\eta}}_{n}=\sum_{\xi=1}^{m} \psi_{n}^{\xi} \mathbf{d}_{n}^{\xi}
$$

\section{Explicit time-stepping scheme}

The procedure implemented into the in-house program is better understood following a quasialgorithmic description. Once the damage is computed for the current time step, the dependent variables and tensors are updated on the elements and, then, they are assembled for the total number of elements on the composite mesh. A detailed description of the FEM operations is clearly out of the main scope of this article. Thus, the discussion is reduced either to the points that differ from the standard scheme due to the proposed damage strategy or to highlight several issues of interest for programming. Readers interested in operations such as assembly or numerical integration are referred to standard textbooks on FEM, such as Hughes (2000) or Zienkiewicz and Taylor (2000), for further details. The algorithm of the explicit time-stepping scheme deployed is provided below in some detail.

(a) initialisation and initial conditions:

- counter and initial time: $n=0, t_{0}=0$,

- initial velocity: $\dot{\mathbf{u}}_{0}=0$,

- initial values of other state variables: $\boldsymbol{\eta}_{0}=0, \boldsymbol{\sigma}_{0}=0$, etc., and

- input nodal coordinates of Lagrangian mesh: $\mathbf{X}_{0}$.

(b) compute lumped-mass matrix $\mathbf{M}$ (Belytschko et al., 2001), which is a diagonal mass matrix in order to have an uncoupled system of equations and hence perform an explicit time-stepping scheme

(c) Loop over time steps:

1. Update configuration by new position of nodal coordinates

$$
\mathbf{X}_{n}=\mathbf{X}_{n-1}+\mathbf{u}_{n}
$$

where $\mathbf{u}_{n}$ denotes the nodal displacements vector.

2. Loop over elements $e$

2.1. Compute strain at current element

$$
\boldsymbol{\varepsilon}_{n}=\mathbf{B} \cdot \mathbf{u}_{n}
$$

2.2. Call to constitutive composite damage law subroutine, flowchart Figure (3), to update material state variables at the element $e$.

$$
\boldsymbol{\varepsilon}_{n}, \boldsymbol{\eta}_{n-1} \longrightarrow \mathbf{C}_{n}, \boldsymbol{\sigma}_{n}, \dot{\boldsymbol{\eta}}_{n}
$$


2.3. Update increment of damage

$$
\Delta \boldsymbol{\eta}_{n}=\Delta t_{n} \dot{\boldsymbol{\eta}}_{n}
$$

2.4. Update damage internal variables

$$
\boldsymbol{\eta}_{n+1}=\boldsymbol{\eta}_{n}+\Delta \boldsymbol{\eta}_{n}
$$

2.5. Compute element nodal internal force vector

$$
\mathbf{f}_{n}^{(e)}=\int_{\Omega_{e}} \mathbf{B}^{T} \cdot \mathbf{C}_{n} \cdot \mathbf{B} \mathrm{d} \Omega
$$

2.6. End loop over elements

3. Global internal force vector, assembly for all elements

$$
\mathbf{f}_{n}^{(i n t)}=\bigwedge_{e=1}^{\text {numel }} \mathbf{f}_{n}^{(e)}
$$

4. If external forces are time-dependent, update nodal external force vector

$$
\mathbf{f}_{n}^{(e x t)}=\bigwedge_{e=1}^{\text {numel }}\left\{\int_{\Omega^{e}} \mathbf{N}^{T} \boldsymbol{b}_{n} \mathrm{~d} \Omega+\int_{\Gamma^{e}} \mathbf{N}^{T} \boldsymbol{q}_{n} \mathrm{~d} \Gamma\right\}
$$

where, $N$ is a tensor containing the shape functions, $\mathbf{b}_{n}$ the body forces, $\mathbf{q}_{n}$ the traction forces applied over the boundary of the body, B is the strains operator (Hughes, 2000), $\mathbf{f}^{(i n t)}\left(\boldsymbol{\eta}_{n}\right)$ the nodal internal forces which are damage dependent and $\mathbf{f}_{n}^{(e x t)}$ the external forces. Counter index is written for the external force vector to indicate a possible variation with the time-stepping scheme. The symbol $\bigwedge_{e=1}^{\text {numel }}$ denotes the assembly for the total number of elements in the mesh numel. The integrals over the finite element domain are approximated by means of numerical integration (Hughes, 2000).

5. Compute acceleration from momentum finite element system of equations

$$
\ddot{\mathbf{u}}_{n}=\mathbf{M}^{-1} \cdot\left[\mathbf{f}_{n}^{(e x t)}-\mathbf{f}^{(i n t)}\left(\boldsymbol{\eta}_{n}\right)\right]
$$

where $\ddot{\mathbf{u}}_{u}$ is the acceleration vector containing the nodal acceleration.

6. Compute the nodal mid-step velocities $\dot{\mathbf{u}}_{n+1 / 2}$ as

$$
\dot{\mathbf{u}}_{n+1 / 2}=\dot{\mathbf{u}}_{n-1 / 2}+\Delta t_{n} \ddot{\mathbf{u}}_{n}
$$

7. Calculate new nodal displacement vector

$$
\mathbf{u}_{n+1}=\mathbf{u}_{n}+\Delta t_{n+1 / 2} \dot{\mathbf{u}}_{n+1 / 2}
$$

(d) calculate new critical time step $\Delta t_{n}^{(\text {crit })}$, update time step $\left.\left.\Delta t_{n}=\alpha \Delta t_{n}^{(\text {crit })}, \alpha \subseteq\right] 0,1\right]$ and check convergence (relative norm of the residual)

(e) end loop over time steps

\section{Numerical results and discussion}

An in-house three-dimensional XPFEM code was developed for the numerical technique. In this manner, the subroutines were written in an accessible environment which demonstrated to be versatile for straightforward modifications. Once this pilot program was conveniently validated, the material subroutines were translated into a VUMAT subroutine within ABAQUS to provide broader postprocessing of results. 


\section{Single element under tensile loading in fibre direction}

In this test, the assessed damage modes were fibre rupture, matrix cracking, fibre kinking and matrix crushing $^{\mathrm{b}}$ (see appendix for the used tensors related to the different damage modes). Obviously, the two latter ones should not appear in a tension test as described here. In tension, the typical modes of failure are fibre rupture and matrix cracking. The model was able to detect only these two modes for tension and therefore simulating only the expected ones.

The numerical technique is validated by comparing the predicted ultimate tensile stress and failure strain for a carbon fibre reinforced finite element with the corresponding values obtained by experimental testing. The experimental material properties were obtained by Soden et al. (1998b). Elastic properties and material strengths used are presented in Table 1.

The finite element is an eight-noded brick element with reduced integration. ${ }^{\mathrm{c}}$ The tensile load was applied incrementally in the longitudinal direction of the fibres, trespassing on failure, and was exerted until the load-bearing capacity of the element vanished. Thus, the different damage modes computed were fully recorded if they were present at any stage of the simulation to validate the process. The load is applied slowly, neglecting inertia and is perpendicular to one of the sides of the element in the longitudinal direction of the fibres. The numerical oscillations associated to $X P F E M$ - and other explicit numerical methods - were attenuated using an artificial damping term in the weak form of the momentum equations, i.e. dynamics relaxation (Belytschko and Hughes, 2001). In this manner, the steady-state solution, i.e. after an initial transient, represents the solution to the quasi-static problem (Curiel Sosa et al., 2006).

The obtained failure parameters, ultimate tensile stress and failure strain correlated well with the experimental ones, as given in Table 2. The results show that the expected damage modes involved in the tensile loading failure, i.e. fibre rupture and matrix cracking, were computed whereas matrix crushing and fibre kinking were not. This highlights the capacity of the technique to detect the correct damage modes associated to a particular stress state.

\section{Shearing on plane 12 of single element}

In this test, the element, with material parameters from Table 1, is subjected to shearing in plane 12. The load was linearly incremented until complete failure by shear. The comparison of numerical and experimental responses are presented in Table 3. The numerical results show a relative error of $19 \%$ in the failure strain, although the averaged relative error remained small. The predicted failure stress was in good agreement with the experimental one.

\section{Single element under compression in fibre direction}

The single element described in 'Single element under tensile loading in fibre direction' is used here with reverse loading, i.e. compression, up to complete failure. The material parameters are the same of those in Table 1. The failure stress is presented in Table 4. Stability was observed during execution of the program until the element completely lost its structural resistance. The damage modes recorded were fibre kinking and matrix crushing. Neither matrix cracking nor fibre rupture was observed in the numerical test.

\footnotetext{
${ }^{\mathrm{b}}$ Other modes may be flexibly introduced in the envisaged framework.

'Reduced integration performs computation of stress, and other variables, in one quadrature point (Belytschko et al., 200I).
} 
Table I. Material properties of the carbon reinforced epoxy lamina (Soden et al., 1998b) used in single-element tests.

\begin{tabular}{lc}
\hline Lamina properties (single-element tests) & 126 \\
\hline Young's modulus, $E_{1}(\mathrm{GPa})$ & $1 \mathrm{I}$ \\
Young's modulus, $E_{2}, E_{3}(\mathrm{GPa})$ & 6.6 \\
Shear modulus, $S_{12}, S_{31}(\mathrm{GPa})$ & 3.93 \\
Shear modulus, $S_{23}(\mathrm{GPa})$ & 0.28 \\
Poisson ratios, $v_{12}, v_{31}$ & 0.4 \\
Poisson ratio, $v_{23}$ & 1950 \\
Tensile strength in fibre direction, $X_{11}^{(t)}(\mathrm{MPa})$ & -1480 \\
Compressive strength in fibre direction, $X_{11}^{(c)}(\mathrm{MPa})$ & 48 \\
Perpendicular to fibre tensile strength, $X_{22}^{(t)}, X_{33}^{(t)}(\mathrm{MPa})$ & -200 \\
Perpendicular to fibre compressive strength, $X_{22}^{(c)}, X_{33}^{(c)}(\mathrm{MPa})$ & 79 \\
Shear strength, $X_{12}, X_{31}(\mathrm{MPa})$ & 17.14 \\
Shear strength, $X_{23}(\mathrm{MPa})$ &
\end{tabular}

Superscripts $(t)$ and $(c)$ denote tension and compression respectively.

Table 2. Values of ultimate stress, $\sigma_{11, U T S}$, and failure strain, $\varepsilon_{11, f}$ from longitudinal (fibre direction) tensile loading tests on a lamina with mechanical properties given in Table I.

\begin{tabular}{llll}
\hline & Experimental $^{\mathrm{a}}$ & Numerical & Relative error (\%) \\
\hline$\sigma_{11, \text { UTS }}(\mathrm{MPa})$ & 1950 & 1966.7 & 0.82 \\
$\varepsilon_{11, f}(\%)$ & 0.0138 & 0.0157 & 14.5 \\
\hline
\end{tabular}

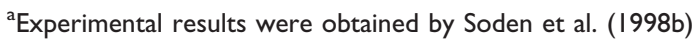

Table 3. Results from shearing on plane 12 of single element.

\begin{tabular}{llll}
\hline & Experimental & Numerical & Relative error (\%) \\
\hline$\sigma_{12, f}(\mathrm{MPa})$ & 79 & 84.3 & 6.7 \\
$\varepsilon_{12, f}(\%)$ & 0.021 & 0.025 & 19.07 \\
\hline
\end{tabular}

Table 4. Results for single element under compression in fibre direction.

\begin{tabular}{llll}
\hline & Experimental & Numerical & Relative error (\%) \\
\hline$\sigma_{1 \mathrm{I}, f}(\mathrm{MPa})$ & -1480 & -1558 & 5.2 \\
\hline
\end{tabular}

\section{Low-velocity impact on laminate}

The proposed technique is used to simulate maps of damage in a cross-ply laminate when impacted by a projectile at low velocity. Hou et al. (2000) performed this test. Their experimental work involved the use of a titanium projectile of $260 \mathrm{~g}, 9.55 \mathrm{~mm}$ in diameter and ended with a spherical 
steel cap $16 \mathrm{~mm}$ in diameter that impacted on a laminate at $7.08 \mathrm{~m} / \mathrm{s}$. Rubber bungs were utilised to attenuate the oscillations of the forced signal in the projectile. The target laminate has a stacking sequence of 21 alternated plies with orientations [0,90] with a percentage of fibre volume of $60 \%$. Plies are made of one layer of UD carbon fibres within an epoxy matrix. The composite material is commonly specified as CFRP T300/914 and its material properties are given in Table 5. The test was carried out by means of a gas gun apparatus (Hou et al., 2000). The projectile is launched by the gas gun and is impacted on the ring-supported laminate, as depicted in Figure 4. The sample is initially unstressed. The dimensions of the laminate were $85 \times 85 \times 2.6 \mathrm{~mm}^{3}$. The support for the specimen is located at the side opposite to the impact and it is a ring of hole diameter $45 \mathrm{~mm}$.

Hou et al. (2000) measured the projectile speed by infrared timing gates immediately before the contact between the projectile and the target. Dye penetrants were utilised to visualise the damage which was recorded by $\mathrm{C}$-scans later on. Perhaps, the most significant result was that delamination

Table 5. Material properties of the lamina used in the manufacturing of the target laminate taken from Hou et al. (2000).

Material properties (low-velocity impact test)

Density, $\rho\left(\mathrm{kg} / \mathrm{m}^{3}\right)$

1580

Young's modulus, $E_{1}$ (GPa)

Young's modulus, $E_{2}, E_{3}$ (GPa)

9.4

Shear modulus, $S_{12}, S_{31}(\mathrm{GPa})$

4.5

Shear modulus, $S_{23}(\mathrm{GPa})$

2.98

Poisson ratio, $v_{12}, v_{13}$

0.209

Poisson ratio, $v_{21}, v_{31}$

0.00141338

Poisson ratio, $v_{23}, v_{32}$

0.33

2070

Tensile strength in fibre direction, $X_{11}^{(t)}(\mathrm{MPa})$

74

Perpendicular to fibre tensile strength, $X_{22}^{(t)}, X_{33}^{(t)}(\mathrm{MPa})$

Perpendicular to fibre compressive strength, $X_{22}^{(c)}, X_{33}^{(c)}(\mathrm{MPa})$

86

Shear strength, $X_{23}(\mathrm{MPa})$

64

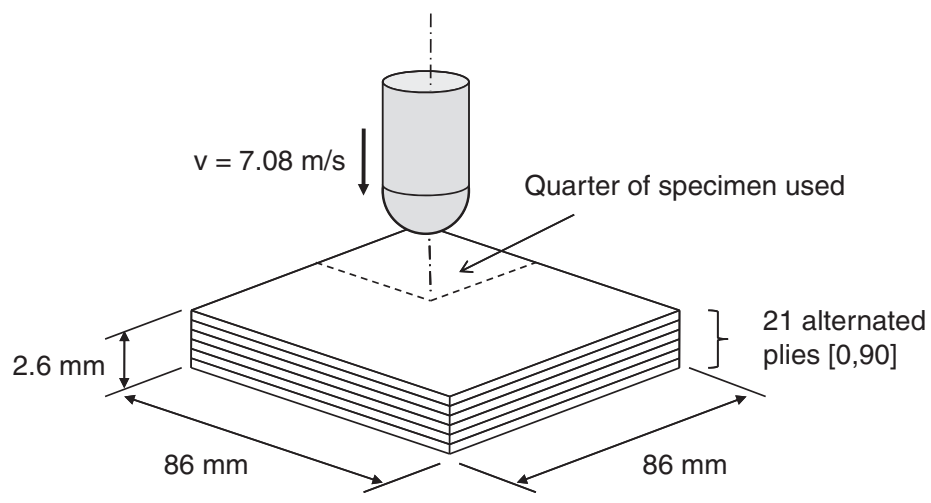

Figure 4. Configuration of the low-velocity impact on target laminate.

The rigid ring support beneath the laminate target is not visible in the perspective shown. 


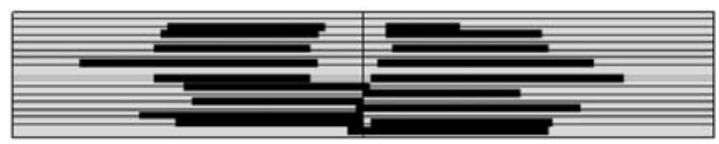

Figure 5. Approximated localisation of delamination from experimental results by Hou et al. (2000). The figure represents a through-thickness view of a quarter of the target laminate. Middle vertical line indicates the corner.

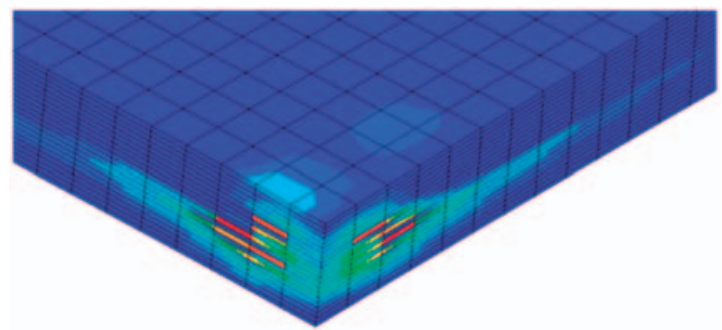

Figure 6. View through section-cut interply damage (delamination) on a quarter laminate. Localisation of delamination correlates well with the experimental map of damage provided by Hou et al. (2000). In that work, a delamination-free region was recorded just beneath the projectile, in through-thickness impact zone. The simulation was able to model that delamination-free zone as can be observed in his figure.

was not observed in the through-thickness compressive region beneath the projectile impact zone, i.e. the centre of the target laminate (Figure 5). Our proposed technique replicates this effect, see Figure 6 . This effect is caused because of the symmetry of the configuration.

The target laminate was modelled using one finite element per ply in the transversal direction, making 21 elements through the thickness of the laminate in total. The finite element used is an eight noded hexahedra element with reduced integration. For this test, the numerical technique was implemented in FORTRAN language into a VUMAT subroutine for ABAQUS software. The number of state variables representing damage was set to six and the number of damage modes modelled was six. These were fibre rupture, fibre kinking, matrix cracking (in both normal-to-fibre directions) and matrix crushing (also in both normal-to-fibre directions). The main expressions of tensors and other variables can be found in 'Appendix'. Thanks to the symmetry and, in order to save computational cost, the configuration was reduced to one quarter of the original geometry for all components, i.e. projectile, specimen and rigid support.

Although the proposed technique is mesh-dependent, regularisation, e.g. Petrinic et al. (2006), is not attempted here as the interest is to replicate experimental response for matrix crushing and delamination mainly with the same mesh as Hou et al. (2000). However, it is encouraged to develop non-local strategies within the proposed technique for simulation of damage independent of the size of the finite elements in future works. Because of the utilisation of reduced integration, hourglassing control $^{\mathrm{d}}$ was used to avoid unrealistic distortion of elements. The contact condition was set for surface-surface. The shear stress fields are depicted in Figures 7 and Figure 8. Shear stresses play a major role in the initiation and development of delamination in these type of problems. Figure 9 shows the final matrix crushing once the projectile has bounced back. The crushing damage is 


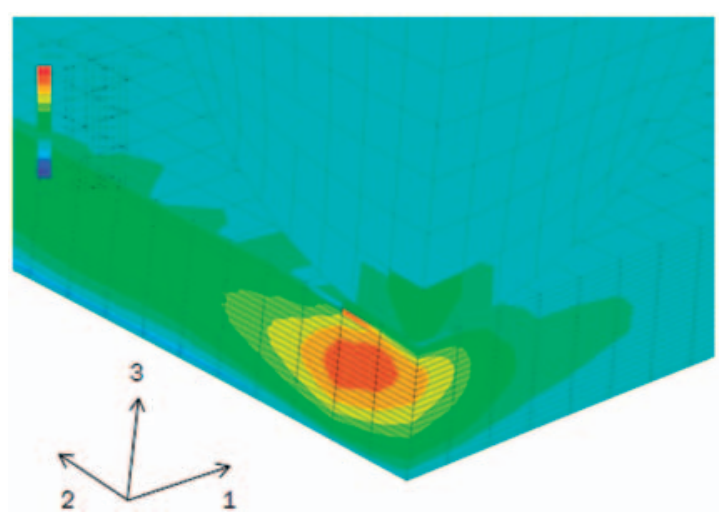

Figure 7. Contour plot of shear stress $\sigma_{23} \cdot \sigma_{23}^{(\text {max })}=36.17 \mathrm{MPa}$ (red contour); $\sigma_{23}^{(\text {min) }}=-15.48 \mathrm{MPa}$ (blue contour).

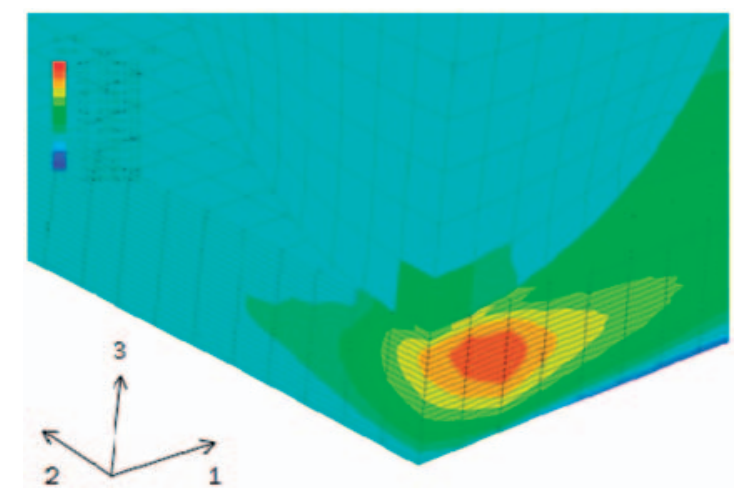

Figure 8. Contour plot of shear stress $\sigma_{13} . \sigma_{13}^{\text {(max) }}=36.15 \mathrm{MPa}$ (red contour); $\sigma_{13}^{(\text {min) }}=-14.76 \mathrm{MPa}$ (blue contour).

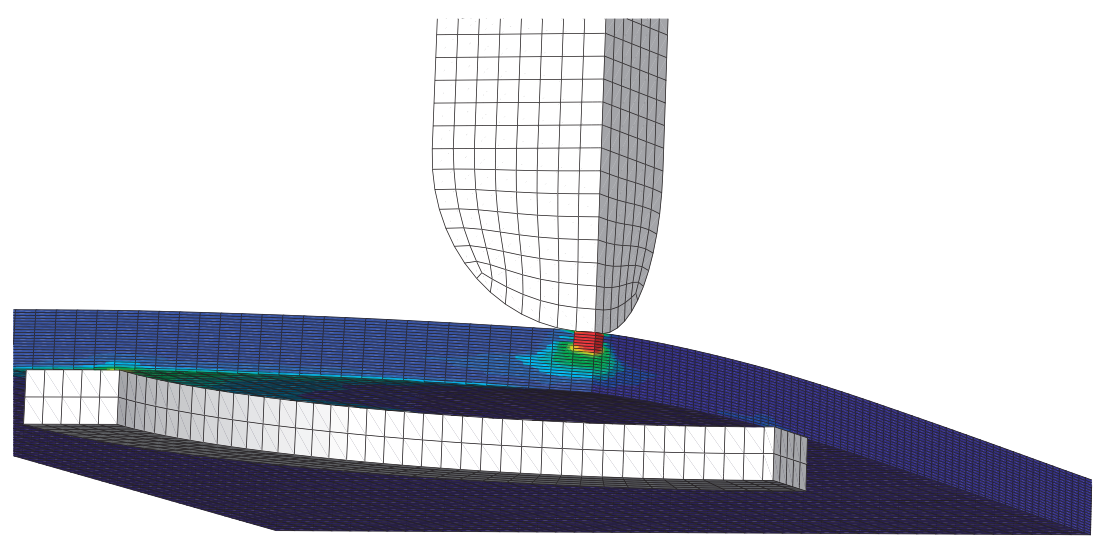

Figure 9. Detail of matrix crushing map of damage in the through-thickness impact zone, under the impactor. The crushed region was observed in the expected location. 
located in the contact zone beneath the impactor which correlates with the results by Hou et al. (2000). Figure 6 represents the delamination region, with grades, matching the experimental map, Figure 5, of damage obtained from the gas gun in a significant region and leaving efficiently without delamination of the central part.

\section{Conclusion}

The development of a new technique for simulation of damage modes in fibre reinforced composite laminates was presented in this article. The explicit time-stepping technique embedded within the FEM was shown in detail as well as novel features, which included:

(a) formulation of damage directions whereby propagation of the different damage modes was computed,

(b) increment of damage, i.e. growth, posed as a function of normalised energy release rates mapped onto strain space for smoother and stable computational executions, and

(c) criteria of damage based on shrinkage of strain damage surfaces and positive accumulation of damage.

Results proved that the proposed technique is able to address the initiation of failure followed by a suddenly disappearing non-linear regime, that eventually precipitates in complete failure. In particular, excellent prediction of delamination was observed in low-velocity impact numerical test, correlating with experiments in great detail.

The evolution of damage is gradually evolving without losing completely the load-bearing capacity of the element once the damage criteria are fulfilled. In this manner, the stability and, therefore, the convergence of the numerical techniques are improved. The technique is sensible to mesh element size, and hence, regularisation techniques are recommended for future works. Furthermore, the use of cohesive elements in conjunction with the technique would possibly provide a more accurate description of discontinuities associated to damage although this has not been attempted at this time.

\section{Funding \\ 4 III.}

\section{References}

Allen DH, Harris C and Groves SE (1987) A thermomechanical constitutive theory for elastic composites with distributed damage. Part I: Theoretical development. International Journal of Solids and Structures 23(9): 1301-1318.

Belytschko T and Hughes TJR (2001) Computational Methods for Transient Analysis. The Netherlands: Elsevier Science B.V.

Belytschko T, Liu WK and Moran B (2001) Nonlinear Finite Elements for Continua and Structures. New York: John Wiley \& Sons.

Camanho PP, Dávila CG and Ambur DR (2001) Numerical simulation of delamination growth in composite materials. NASA/TP-2001-211041, Hampton, VA.

Camanho PP and Mathews FL (1999) A progressive damage model for mechanically fastened joints in composite laminates. Journal of Composite Materials 33(24): 2248-2280.

Camanho PP, Dávila CG and de Moura MF (2003) Numerical simulation of mixed-modes progressive delamination in composite materials. Journal of Composite Materials 37(16): 1415-1438. 
Chaboche J-L (1981) Continuous damage mechanics - a tool to describe phenomena before crack initiation. Nuclear Engineering and Design 64: 233-247.

Chaboche J-L (1992) Damage induced anisotropy: on the difficulties associated with the active/passive unilateral condition. International Journal of Damage Mechanics 1: 148-171.

Chang F-K and Chang KY (1987) A progressive damage model for laminated composites containing stress concentration. Journal of Composite Materials 21: 834-855.

Curiel Sosa JL, de Souza Neto EA and Owen DRJ (2006) A combined implicit-explicit algorithm in time for non-linear finite element analysis. Communications in Numerical Methods Engineering 22: 63-75.

Daniel IM (2007) Failure of composite materials. Strain 43: 4-12.

Desmorat R, Gatuingt F and Ragueneau F (2010) Nonstandard thermodynamics framework for robust computations with induced anisotropic damage. International Journal of Damage Mechanics 19(1): 53-73.

Donadon MV, Iannucci L, Falzon BG, et al. (2008) A progressive failure model for composite laminates subjected to low velocity impact damage. Computers and Structures 86: 1232-1252.

Germain P, Nguyen QS and Suquet P (1983) Continuous thermodynamics. Journal of Applied Mechanics, ASME 50: 1010-1020.

Hallet SR (1997) Small specimen impact testing and modelling of carbon fibre T300/914. PhD Thesis. University of Oxford, UK.

Harris CE, Coats TW, Allen DH, et al. (1995) A progressive damage model and analysis methodology for predicting the residual strength of composite laminates. Journal of Composites Technology and Research 29: 926-981.

Hashin Z (1980) Failure criteria for unidirectional fiber composites. Journal of Applied Mechanics 47: 329-334.

Hinton MJ and Soden PD (1998) Predicting failure in composite laminates, the background to the exercise. Composites Science and Technology 58: 1001-1010.

Hou JP, Petrinic N, Ruiz C and Hallet SR (2000) Prediction of impact damage in composite plates. Composites Science and Technology 60: 273-281.

Hughes TJR (2000) The Finite Element Method. Linear Static and Dynamic Finite Element Analysis. New York: Dover.

Iannucci L and Willows ML (2006) An energy based damage mechanics approach to modelling impact onto woven composite materials. Part I: Numerical models. Composites: Part A 37: 2041-2056.

Johnson AF, Pickett AK and Rozycki P (2001) Computational methods for predicting impact damage in composite structures. Composites Science and Technology 61: 2183-2192.

Lemaitre J and Chaboche JL (1990) Mechanics of Solids Materials. Cambridge: Cambridge University Press.

Liu PF and Zheng JY (2010) Recent developments on damage modeling and finite element analysis for composite laminates: a review. Materials and Design 31: 3825-3834.

Lundmark P and Varna $\mathbf{J}$ (2005) Constitutive relationships for laminates with ply cracks in inplane loading. International Journal of Damage Mechanics 14: 235-259.

Maimi P, Camanho PP, Mayugo JA, et al. (2007a) A continuum damage model for composite laminates: Part I - Constitutive model. Mechanics of Materials 39(10): 897-908.

Maimi P, Camanho PP, Mayugo JA, et al. (2007b) A continuum damage model for composite laminates: Part II - Computational implementation and validation. Mechanics of Materials 39(10): 909-919.

Matzenmiller A, Lubliner J and Taylor RL (1995) A constitutive model for anisotropic damage in fibercomposites. Mechanics of Materials 20: 125-152.

Petrinic N, Curiel Sosa JL, Siviour CR, et al. (2006) Improved predictive modelling of strain localisation and ductile fracture in a Ti-6Al-4V alloy subjected to impact loading. Journal of Physics IV 134: $147-155$.

Puck A and Schurmann H (1998) Failure analysis of FRP laminates by means of physically based phenomenological models. Composites Science and Technology 58: 1045-1067.

Raimondo L, Iannucci L, Robinson P, et al. (2012) A progressive failure model for mesh-size-independent FE analysis of composite laminates subject to low-velocity impact damage. Composites Science and Technology 72: 624-632. 
Simo JC and Ju JW (1987) Strain- and stress-based continuum damage models - I. Formulation. International Journal of Solids and Structures 23(7): 821-840.

Singh CV and Talreja R (2008) Analysis of multiple off-axis ply cracks in composite laminates. International Journal of Solids and Structures 45(16): 4574-4589.

Singh CV and Talreja R (2010) Evolution of ply cracks in multidirectional composite laminates. International Journal of Solids and Structures 47(10): 1338-1349.

5

Soden PD, Hinton MJ and Kaddour AS (1998a) A comparison of the predictive capabilities of current failure theories for composite laminates. Composites Science and Technology 58(7): 1225-1254.

Soden PD, Hinton MJ and Kaddour AS (1998b) Lamina properties, lay-up configurations and loading conditions for a range of fibre-reinforced composite laminates. Composites Science and Technology 58(7): $1011-1022$.

Staab GH (1999) Laminar Composites. Oxford: Butterworth-Heinemann.

Talreja R (1987) Modelling of damage development in composites using internal variables concepts. Proceedings of the ASME, Damage Mechanics in Composites, AD 12: 11-16.

Talreja R (2006) Damage analysis for structural integrity and durability of composite materials. Fatigue and Fracture of Engineering Materials and Structures 29: 481-506.

Tan SC (1991) A progressive failure model for composite laminates containing openings. Journal of Composite Materials 25(5): 556-577.

Tsai SW and Wu EM (1971) General theory of strength for anisotropic materials. Journal of Composite Materials 5: 58-80.

Turon A, Camanho PP, Costa J, et al. (2006) A damage model for the simulation of delamination in advanced composites under variable-mode loading. Mechanics of Materials 38(11): 1072-1089.

Tvergaard V (2004) Predictions of mixed mode interface crack growth using a cohesive zone model for ductile fracture. Journal of Mechanics and Physics of Solids 52(4): 925-940.

Wisnom MR (2010) Modelling discrete failures in composites with interface elements. Composites Part A: Applied Science and Manufacturing 41(7): 795-805.

Yang Q, Zhou W-Y and Chen X (2010) Thermodynamic significance and basis of damage variables and equivalences. International Journalof Damage Mechanics 19(08): 898-910.

Zienkiewicz OC and Taylor RL (2000) The Finite Element Method: The Basis, 1. 5th edn. Oxford: ButterworthHeinemann.

\section{Appendix}

\section{Second-order tensors}

\section{'Damaged' stiffness matrix in orthotropic directions for a lamina}

$$
\begin{aligned}
& C(\eta)=\left[\begin{array}{cccccc}
\frac{\left(1-\eta_{11}\right)\left(1-v_{23} \nu_{32}\right)}{E_{22} E_{33} \gamma} & \frac{\left(1-\eta_{11}\right)\left(\nu_{12}+\nu_{32} \nu_{13}\right)}{E_{11} E_{33} \gamma} & \frac{\left(1-\eta_{11}\right)\left(\nu_{13}+\nu_{12} \nu_{23}\right)}{E_{11} E_{22} \gamma} & 0 & 0 & 0 \\
\frac{\left(1-\eta_{22} 2\right)\left(\nu_{12}+\nu_{32} \nu_{13}\right)}{E_{11} E_{33} \gamma} & \frac{\left(1-\eta_{22}\right)\left(1-v_{13} \nu_{31}\right)}{E_{11} E_{33} \gamma} & \frac{\left(1-\eta_{22}\right)\left(\nu_{23}+\nu_{21} \nu_{13}\right)}{E_{11} E_{22} \gamma} & 0 & 0 & 0 \\
\frac{\left(1-\eta_{33}\right)\left(\nu_{13}+\nu_{12} \nu_{23}\right)}{E_{11} E_{22} \gamma} & \frac{\left(1-\eta_{33}\right)\left(v_{23}+\nu_{21} \nu_{13}\right)}{E_{11} E_{22} \gamma} & \frac{\left(1-\eta_{33}\right)\left(1-\nu_{12} \nu_{21}\right)}{E_{11} E_{22} \gamma} & 0 & 0 & 0 \\
0 & 0 & 0 & \left(1-\eta_{12} S_{12}\right) & 0 & 0 \\
0 & 0 & 0 & 0 & \left(1-\eta_{23} S_{23}\right) & 0 \\
0 & 0 & 0 & 0 & 0 & \left(1-\eta_{31} S_{31}\right)
\end{array}\right] \\
& \gamma=\frac{\left(1-v_{12} v_{21}-v_{23} v_{32}-v_{31} v_{13}-2 v_{21} v_{32} v_{13}\right)}{E_{11} E_{22} E_{33}}
\end{aligned}
$$


Second-order tensors used in the tests for distinct damage modes $(m=6)$

Superscript index denotes damage mode. The tensors evolve with time as internal damage variables change. Thus, the subscript $n$ - time step counter - is written to emphasise this time dependence.

- Fibre rupture or breakage $(\xi=1)$

$$
\mathbf{F}_{n}^{1}=\left[\begin{array}{cccccc}
1 /\left(2 E_{1}\left(1-\eta_{11}^{2}\right) X_{11}^{(t)}\right) & 0 & 0 & 0 & 0 & 0 \\
0 & 0 & 0 & 0 & 0 & 0 \\
0 & 0 & 0 & 0 & 0 & 0 \\
0 & 0 & 0 & 1 /\left(2 S_{12}\left(1-\eta_{12}^{2}\right) X_{12}\right) & 0 & 0 \\
0 & 0 & 0 & 0 & 0 & 0 \\
0 & 0 & 0 & 0 & 0 & 1 /\left(2 E_{31}\left(1-\eta_{31}^{2}\right) X_{31}\right)
\end{array}\right]_{n}
$$

- Fibre kinking $(\xi=2)$

$$
\mathbf{F}_{n}^{2}=\left[\begin{array}{cccccc}
1 /\left(2 E_{1}\left(1-\eta_{11}^{2}\right) X_{11}^{(c)}\right) & 0 & 0 & 0 & 0 & 0 \\
0 & 0 & 0 & 0 & 0 & 0 \\
0 & 0 & 0 & 0 & 0 & 0 \\
0 & 0 & 0 & 1 /\left(2 S_{12}\left(1-\eta_{12}^{2}\right) X_{12}\right) & 0 & 0 \\
0 & 0 & 0 & 0 & 0 & 0 \\
0 & 0 & 0 & 0 & 0 & 1 /\left(2 E_{31}\left(1-\eta_{31}^{2}\right) X_{31}\right)
\end{array}\right]_{n}
$$

- Interply damage, mode II ( $\xi=3)$

$$
\mathbf{F}_{n}^{3}=\left[\begin{array}{cccccc}
0 & 0 & 0 & 0 & 0 & 0 \\
0 & 1 /\left(2 E_{2}\left(1-\eta_{22}^{2}\right) X_{22}^{(t)}\right) & 0 & 0 & 0 & 0 \\
0 & 0 & 0 & 0 & 0 & 0 \\
0 & 0 & 0 & 1 /\left(2 E_{12}\left(1-\eta_{12}^{2}\right) X_{12}\right) & 0 & 0 \\
0 & 0 & 0 & 0 & 1 /\left(2 E_{23}\left(1-\eta_{23}^{2}\right) X_{23}\right) & 0 \\
0 & 0 & 0 & 0 & 0 & 0
\end{array}\right]_{n}
$$

- Matrix crushing in 2-direction - in-plane perpendicular to fibre - $(\xi=4)$

$$
\mathbf{F}_{n}^{4}=\left[\begin{array}{cccccc}
0 & 0 & 0 & 0 & 0 & 0 \\
0 & 1 /\left(2 E_{2}\left(1-\eta_{22}^{2}\right) X_{22}^{(c)}\right) & 0 & 0 & 0 & 0 \\
0 & 0 & 0 & 0 & 0 & 0 \\
0 & 0 & 0 & 1 /\left(2 E_{12}\left(1-\eta_{12}^{2}\right) X_{12}\right) & 0 & 0 \\
0 & 0 & 0 & 0 & 1 /\left(2 E_{23}\left(1-\eta_{23}^{2}\right) X_{23}\right) & 0 \\
0 & 0 & 0 & 0 & 0 & 0
\end{array}\right]_{n}
$$


- Interply damage, mode I $(\xi=5)$

$$
\mathbf{F}_{n}^{5}=\left[\begin{array}{cccccc}
0 & 0 & 0 & 0 & 0 & 0 \\
0 & 0 & 0 & 0 & 0 & 0 \\
0 & 0 & 1 /\left(2 E_{3}\left(1-\eta_{33}^{2}\right) X_{33}^{(t)}\right) & 0 & 0 & 0 \\
0 & 0 & 0 & 0 & 0 & 0 \\
0 & 0 & 0 & 0 & 1 /\left(2 E_{23}\left(1-\eta_{23}^{2}\right) X_{23}\right) & 1 /\left(2 E_{31}\left(1-\eta_{31}^{2}\right) X_{31}\right)
\end{array}\right]_{n}
$$

- Matrix crushing in 3-direction through thickness, i.e. out-of-plane perpendicular to fibres $(\xi=6)$

$$
\mathbf{F}_{n}^{6}=\left[\begin{array}{cccccc}
0 & 0 & 0 & 0 & 0 & 0 \\
0 & 0 & 0 & 0 & 0 & 0 \\
0 & 0 & 1 /\left(2 E_{3}\left(1-\eta_{33}^{2}\right) X_{33}^{(c)}\right) & 0 & 0 & 0 \\
0 & 0 & 0 & 0 & 0 & 0 \\
0 & 0 & 0 & 0 & 1 /\left(2 E_{23}\left(1-\eta_{23}^{2}\right) X_{23}\right) & 0 \\
0 & 0 & 0 & 0 & 0 & 1 /\left(2 E_{31}\left(1-\eta_{31}^{2}\right) X_{31}\right)
\end{array}\right]_{n}
$$

